\title{
A GENERALIZATION OF WARING'S THEOREM ON NINE CUBES*
}

BY L. E. DICKSON

TheOREM. Every positive integer $p$ can be expressed as a sum of seven cubes and the double of a cube, the cubes being positive or zero integers.

On the basis of known tables, this theorem holds for $p \leqq 40,000$ as shown by the writer in the American Mathematical Monthly for April, 1927. The further empirical theorems of that paper will not be discussed here.

We shall here prove the above theorem for all sufficiently large integers $p$. The proof is analogous to that employed by Landau $\dagger$ in proving his theorem that every sufficiently large integer is a sum of at most eight positive integral cubes.

Let $r$ be the real ninth root of $4 / 3$. The number of the primes $\equiv 2(\bmod 3)$ which exceed $x$ and are $\leqq r x$ is known to increase indefinitely with $x$. As $x$ we choose the first radical in (1). Hence for all sufficiently large integers $n$, there exist at least ten primes $p$ such that

$$
\begin{gathered}
(n / 12)^{1 / 9}<p \leqq(n / 9)^{1 / 9}, \\
p \equiv 2(\bmod 3) .
\end{gathered}
$$

The product of the ten primes exceeds $(n / 12)^{10 / 9}$ and hence exceeds $n$ if $n>12^{10}$. Hence not all of the ten are divisors of $n$.

To give a numerical illustration, take $n=9 \mathrm{~m}^{9}$. Then (1) becomes $m / r<p \leqq m$. For $m=6000, m / r=5811.2$, and the primes $p$ satisfying also (2) are

* Presented to the Society, April 15, 1927.

$\dagger$ Mathematische Annalen, vol. 66 (1909), pp. 102-5. Reproduced in his Verteilung der Primzahlen, vol. 1, 1909, pp. 555-59. 


$$
\begin{array}{llllll}
5813, & 5843, & 5849, & 5861, & 5867, & 5879, \\
5897, & 5903, & 5927, & 5939, & 5981, & 5987 .
\end{array}
$$

The third prime just exceeds $m^{\prime} / r$ when $m^{\prime}=6038$. Hence the last ten primes of our list serve for every $m$ between 6000 and 6038 inclusive.

Let therefore $p$ be a prime not dividing $n$ such that (1) and (2) hold. Then

$$
9 p^{9} \leqq n<12 p^{9}
$$

A slight modification of Landau's proof of his first lemma shows that if $p$ is an odd prime satisfying (2), every integer not divisible by $p$ is congruent modulo $p^{3}$ to the double of a cube. Hence there are integers $\delta$ and $M$ satisfying

$$
n-2 \delta^{3}=p^{3} M, \quad 0<\delta<p^{3} .
$$

Then

$9 p^{9}-2 p^{9} \leqq n-2 p^{9}<n-2 \delta^{3}=p^{3} M, \quad p^{3} M<n<12 p^{9}$.

Cancelling the factors $p^{3}$, we have

$$
7 p^{6}<M<12 p^{6} .
$$

The further discussion by Landau* applies here unchanged and shows that $n$ is the sum of $2 \delta^{3}$ and seven integral cubes $\geqq 0$. We may replace $2 \delta^{3}$ by $k \delta^{3} ; k \geqq 1$.

The University of Chicago

* His condition for $M_{2}>0$ is satisfied if $p \geqq 10$. Since the largest $\gamma$ is 22 , we obtain the milder condition $p \geqq 5$. Hence $n$ exceeds 13 million. 